\title{
Abnormal expression of miR-4784 in chondrocytes of osteoarthritis and associations with chondrocyte hyperplasia
}

\author{
JING LIU $^{1 *}$, QIAOLONG YU ${ }^{2 *}$, YANHUI YE ${ }^{2}$, YAN YAN $^{2}$ and XIN CHEN ${ }^{2}$ \\ Departments of ${ }^{1}$ Nosocomial Infection Control and ${ }^{2}$ General Dentistry, The 174th Hospital of Chinese PLA, \\ Chenggong Hospital Affiliated to Medical College of Xiamen University, Xiamen, Fujian 361003, P.R. China
}

Received January 29, 2018; Accepted September 4, 2018

DOI: $10.3892 / \mathrm{etm} .2018 .6739$

\begin{abstract}
The aim of the present study was to assess the expression of microRNA (miRNA)-4784 in the chondrocytes of early osteoarthritis (OA) and to determine the effect of double-stranded (ds)-miRNA-4784 transfection on chondrocyte function. Following the construction of an OA rabbit model, normal chondrocytes (normal control group), OA chondrocytes obtained 4 weeks after modeling (OA at week 4 group) and 8 weeks after modeling (OA at week 8 group) were used. The relative expression of miRNA-4784 in each group was detected using reverse transcription-quantitative polymerase chain reaction (RT-qPCR). Western blotting was performed to measure the expression of type II collagen (Col2a1) and matrix metalloproteinase (MMP)-3 in each group with or without ds-miRNA-4784 transfection. The results revealed that the levels of miR-4784 in groups OA at week 4 and 8 were significantly lower than that of normal control group $(\mathrm{P}<0.05)$. It was also demonstrated that Col2a1 mRNA expression levels in groups OA at week 4 and 8 were 49 and $38 \%$ of that in the normal control group, respectively. Furthermore, MMP-3 mRNA expression levels increased by 3.12- and 3.95-fold in groups OA at week 4 and 8 , respectively, compared with those in the normal control group $(\mathrm{P}<0.01)$. Following transfection with ds-miRNA-4784, Col2al mRNA expression levels increased by 63 and $126 \%$ compared with the levels prior to treatment in groups $\mathrm{OA}$ at week 4 and 8 , respectively $(\mathrm{P}<0.01)$. The expression levels of MMP-3 mRNA in groups OA at week 4 and 8 decreased following transfection compared with the levels prior to treatment. Col2a1 and MMP-3 protein expression exhibited similar patterns to the mRNA expression. In summary, the results of the present study suggest
\end{abstract}

Correspondence to: Dr Xin Chen, Department of General Dentistry, The 174th Hospital of Chinese PLA, Chenggong Hospital Affiliated to Medical College of Xiamen University, 92-96 Wenyuan Road, Xiamen, Fujian 361003, P.R. China

E-mail:chr3cf@163.com

*Contributed equally

Key words: chondrocyte hyperplasia, microRNA-4784, osteoarthritis, transfection, western blotting that miRNA-4784 expression is significantly reduced in early stage OA chondrocytes. Transfection with ds-miRNA-4784 promotes the expression of Col2al and inhibits the MMP-3 expression in chondrocytes.

\section{Introduction}

Osteoarthritis $(\mathrm{OA})$ is a disease characterized by progressive chondrocyte degeneration, synovial hyperplasia in joints, narrowing of the joint space and dysregulation of extracellular matrix metabolism (1). OA primarily affects the knee, hip and shoulder joints and the main symptoms include joint pain, swelling, joint deformity and limited mobility (2). The pathogenesis of $\mathrm{OA}$ is associated with aging, obesity, inflammation, immunity, genetics and many other factors (3). Cartilage degeneration is considered to be one of the primary pathological changes that cause OA, and this typically occurs due to metabolic disorders of extracellular matrix synthesis and degradation (4). Chondrocytes, as the primary cell type found in cartilage tissue, serve important roles in the maintenance of bone and joint structure and function $(5,6)$. Previous studies have demonstrated that matrix metalloproteinases (MMPs) and collagen also serve an important role in the development of OA $(7,8)$. MMPs are ion-active proteases that are ubiquitous in the cartilage and function to degenerate chondrocytes and degrade the extracellular matrix in the cartilage (9-11). MicroRNAs (miRNAs) are single-stranded non-coding RNAs that are associated with the development and progression of OA (12). It has been reported that miRNAs serve a role in the pathogenesis of OA regulating the expression of inflammatory mediators, vascular endothelial growth factor and nerve growth factor (12). Further studies have revealed that miRNAs may inhibit or promote the expression of MMPs and collagen, resulting in the degeneration of chondrocytes and cartilage extracellular matrix, eventually leading to OA $(13,14)$.

Previous studies have also reported that miRNA-4784 expression is downregulated in certain types of cancer, including breast and liver cancer, and miRNA-4784 is involved in the development and progression of cancer by affecting the Akt signal pathway $(12,13)$. To the best of our knowledge, no previous studies have assessed the expression and mechanism of action of miRNA-4784 in OA. The aim of the present study was to assess the expression of miRNA-4784 in OA chondrocytes and the effect of transfection with exogenous 
double-stranded (ds)-miRNA-4784 on the chondrocyte function. The results of the present study may provide a theoretical basis for a novel treatment of OA.

\section{Materials and methods}

Experimental reagents. Lipofectamine ${ }^{\circledR} 2000$, Dulbecco's modified Eagle's medium (DMEM), PBS and penicillin were purchased from Gibco (Gibco; Thermo Fisher Scientific, Inc., Waltham, MA, USA). The miR-4784 mimic was purchased from GenePharma Co., Ltd. (Shanghai, China). Fetal bovine serum and western blotting reagents (cat no. YJ1012465) were purchased from Yiji (Shanghai, China) and radioimmunoprecipitation cell lysis solutions from Beyotime Institute of Biotechnology (Haimen, China). Cell culture plates, Col2a1 and MMP-3 rat anti-rabbit monoclonal antibodies were purchased from Pierce (cat nos. ab185430 and ab26111; Pierce; Abcam, Shanghai, China). The quantitative polymerase chain reaction (qPCR) kit and the reverse transcription (RT) kit were purchased from Fermentas (Thermo Fisher Scientific, Inc.).

Experimental animals and OA model establishment. A total of 40 New Zealand rabbits (age, 6 months; 20 male and 20 female; weight, 2.0-3.0 kg) were purchased from the West China Center of Medical Sciences, Sichuan University (Chengdu, China). The rabbits were raised with controlled temperature and light cycles $\left(24^{\circ} \mathrm{C}\right.$ and $12 / 12$ light cycles) and free access to water and food. The humidity was $60 \pm 10 \%$ for 2 weeks prior to surgery. The following method was used to establish the adult rabbit OA model: pentobarbital sodium $(3 \% ; 30 \mathrm{mg} / \mathrm{kg})$ was injected into the ear vein for anesthesia, and then the rabbits were fixed on an operating table in the supine position. The right knee was shaved and disinfected with iodophor (Abcam). The medial patella meniscus was opened and the patella was everted. The knee joint was then buckled and the anterior segment ligament and collateral ligament were cut with an ophthalmic clip. The surgical site was rinsed, the joint capsule was closed and the skin was disinfected. The right limb remained unfixed and rabbits were kept in a separate case, and had free access to water and food until they were sacrificed. A daily intramuscular injection of $80 \times 10^{6} \mathrm{U}$ of penicillin was administered for 1 week. Right knee tissues were collected at 4 and 8 weeks following modeling to serve as groups OA at week 4 and 8 , respectively (each, $n=10$ ). The left knee of rabbit without treatment was set as control group $(n=10)$. The present study was approved by the Ethics Committee of the 174th Hospital of Chinese PLA (Chenggong Hospital Affiliated to Medical College of Xiamen University, Xiamen, China).

Cell culture. Rabbits were sacrificed at week 4 and 8 following surgery in groups OA at week 4 and 8, respectively. Aseptic cartilage specimens were obtained under sterile conditions. Cartilage tissues with a thickness of 1-2 mm were scraped using a surgical blade and digested with $0.25 \%$ trypsin for $30 \mathrm{~min}$ at $24^{\circ} \mathrm{C}$. Tissues were then digested with $0.2 \%$ collagen II protease for $2 \mathrm{~h}$ at $37^{\circ} \mathrm{C}$. This procedure was repeated 2-3 times to collect chondrocytes, which were subsequently cultured in DMEM supplemented with $10 \%$ fetal bovine serum and $100 \mathrm{U} / \mathrm{ml}$ penicillin at $37^{\circ} \mathrm{C}$ and $5 \% \mathrm{CO}_{2}$.
Transfection of ds-miRNA-4784 into OA chondrocytes. Cells were transfected with ds-miRNA-4784 (UGAGGAGAU GCUGGGACUGA; Thermo Fisher Scientific, Inc.) at $100 \mathrm{nM}$ in accordance with the manufacturer's instructions (Guangzhou RiboBio Co., Ltd., Guangzhou, China). Chondrocytes were cultured overnight to reach a cell density of $40-50 \%$ at $37^{\circ} \mathrm{C}$. The miRNA-4784 mimic was then transfected into chondrocytes using Lipofectamine ${ }^{\circledR} 2000$ for $6 \mathrm{~h}$ at $37^{\circ} \mathrm{C}$. Fresh DMEM was then added, and then the cells were cultured at $37^{\circ} \mathrm{C}$ and $5 \% \mathrm{CO}_{2}$. Cells were harvested at 48-96 $\mathrm{h}$ after transfection and RT-qPCR was used to detect mRNA expression.

Detection of miRNA and mRNA expression using RT-qPCR. U6 snRNA was used as an endogenous control to quantify the expression of miRNA-4784. The expression of Col2a1 and MMP-3 was quantified using $\beta$-actin as an endogenous control before and after transfection. Total RNA was extracted from OA chondrocytes using TRIzol (Thermo Fisher Scientific, Inc.) and was reverse transcribed into cDNA using the RT kit according to the manufacturer's instructions $\left(37^{\circ} \mathrm{C}\right.$ for $15 \mathrm{~min}, 95^{\circ} \mathrm{C}$ for $5 \mathrm{~min}$ ). PCR thermocycling conditions were as follows: $94^{\circ} \mathrm{C}$ for $2 \mathrm{~min}$, followed by 50 cycles of $94^{\circ} \mathrm{C}$ for $30 \mathrm{sec}, 55^{\circ} \mathrm{C}$ for $30 \mathrm{sec}$ and $72^{\circ} \mathrm{C}$ for $30 \mathrm{sec} . \mathrm{Cq}$ values were processed by $2^{-\Delta \Delta \mathrm{Cq}}$ method and the relative expression of each gene was normalized to their corresponding endogenous controls (15). U6 forward, 5'-TGC GGG TCG TGG CTT CGG CAG-3' and reverse, 5'-CCA GTG CAG GGT CCG AGG-3'; $\beta$-actin forward, 5'-GCTGCGTGTGGCCCCTGAG-3' and reverse, 5'-ACGCAGGATGGCATGAGGGA-3'; Col2a1 forward, 5'-TCC TAA GGG TGC CAA TGG TGA-3' and reverse, 5'-AGG ACC AAC TTT GCC TTGAGG AC-3; MMP-3 forward, 5'-ATTCCATGGAGCCAGGCTTTC-3' and reverse, 5'-CATTTGGGTCAAACTCCAACTGTG-3'.

Detection of Col2al and MMP-3 protein expression using western blotting (cat. no. YJ1012465; Yiji). Cultured chondrocytes were rinsed with PBS and lysed using cell lysis solution for $30 \mathrm{~min}$. Cell lysate was then transferred into a tube and centrifuged at $9,800 \times \mathrm{g}$ for $20 \mathrm{~min}$ at $4^{\circ} \mathrm{C}$ to collect cell supernatant. Total protein concentration was determined using a BCA assay. Protein samples ( $90 \mu \mathrm{g}$ per lane) were separated by $10 \%$ SDS-PAGE and transferred onto polyvinylidene membranes. The membranes were then blocked using $5 \%$ bovine serum albumin (Abcam) at $24^{\circ} \mathrm{C}$ for $2 \mathrm{~h}$. Membranes were washed 3 times with TBST and incubated with Col2a1 rat anti-rabbit monoclonal antibodies (1:500) or MMP-3 rat anti-rabbit monoclonal antibodies (1:500) overnight at $4{ }^{\circ} \mathrm{C}$. After further washes with TBST 3 times, the membranes were incubated with goat anti-rat horseradish peroxidase-labeled secondary polyclonal antibody (1:1,000; cat. no. 7077; Cell Signaling Technology, Danvers, MA, USA) for $1 \mathrm{~h}$ at room temperature. Membranes were washed 3 more times with TBST and ECL reagent (Beyotime, Shanghai, China) was added for signal development. The relative expression level of each protein was normalized to the endogenous control ( $\beta$-actin; 1:1,000, cat. no. 60008-1-Ig, Proteintech, Wuhan, China) using ImageJ 1.48 (NIH, Bethesda, MD, USA).

Statistical analysis. SPSS 13.0 software (SPSS, Inc., Chicago, IL, USA) was used for all statistical analyses and data are 


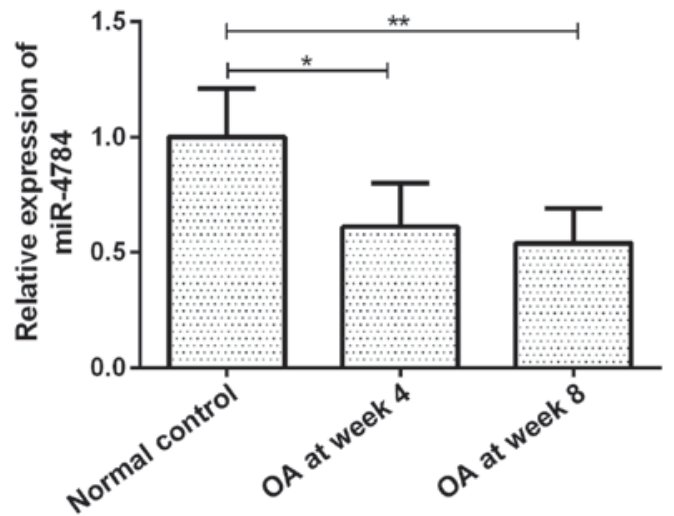

Figure 1. miRNA-4784 expression in normal chondrocytes and OA model chondrocytes at week 4 and 8 after modeling. Data are presented as the mean \pm standard error of the mean. $\mathrm{n}=10 .{ }^{* *} \mathrm{P}<0.01$ and ${ }^{*} \mathrm{P}<0.05$. miRNA, microRNA; OA, osteoarthritis.

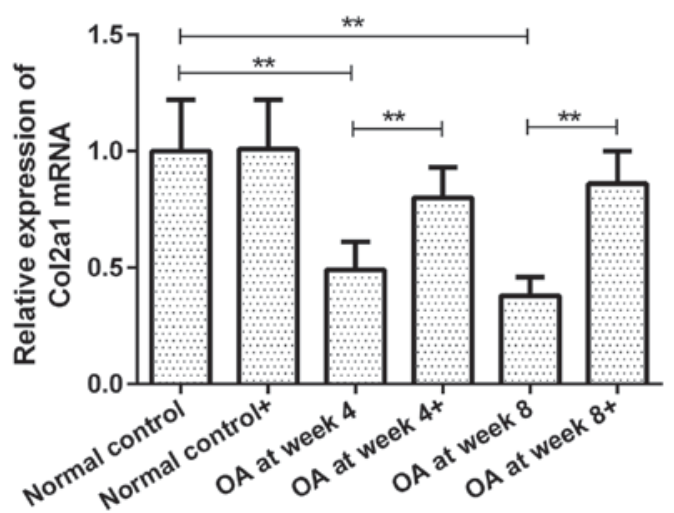

Figure 2. Col2a1 mRNA expression in chondrocytes. Shown are normal chondrocytes and chondrocytes at week 4 and 8 after the establishment of the OA model, respectively. + indicates chondrocytes following transfection. Data are presented as the mean \pm standard error of the mean. $n=10 .{ }^{* *} \mathrm{P}<0.01$. OA, osteoarthritis.

expressed as the mean \pm standard error of the mean. One-way analysis of variance was used for multiple group comparisons and a post-hoc Dunnett's test was performed. $\mathrm{P}<0.05$ was considered to indicate a statistically significant difference.

\section{Results}

miR-4784 expression. The expression of miR-4784 in OA articular chondrocytes gradually decreased with disease progression. miR-4784 expression in groups OA at week 4 and 8 was $61(\mathrm{P}<0.05)$ and $54 \%(\mathrm{P}<0.01)$ of that in the group normal control, respectively (Fig. 1). However, no significant difference in miR-4784 expression was observed between groups OA at week 4 and 8 (Fig. 1).

Expression of Col2al mRNA in chondrocytes prior to and following ds-miRNA-4784 transfection. Col2a1 mRNA expression in groups $\mathrm{OA}$ at week 4 and 8 prior to transfection were 49 and $38 \%$ of that in normal control group, respectively $(\mathrm{P}<0.01$; Fig. 2). However, no significant difference was observed between groups OA at week 4 and 8. Following ds-miRNA-4784 transfection (ds-miRNA-4784+), no significant change in Col2al
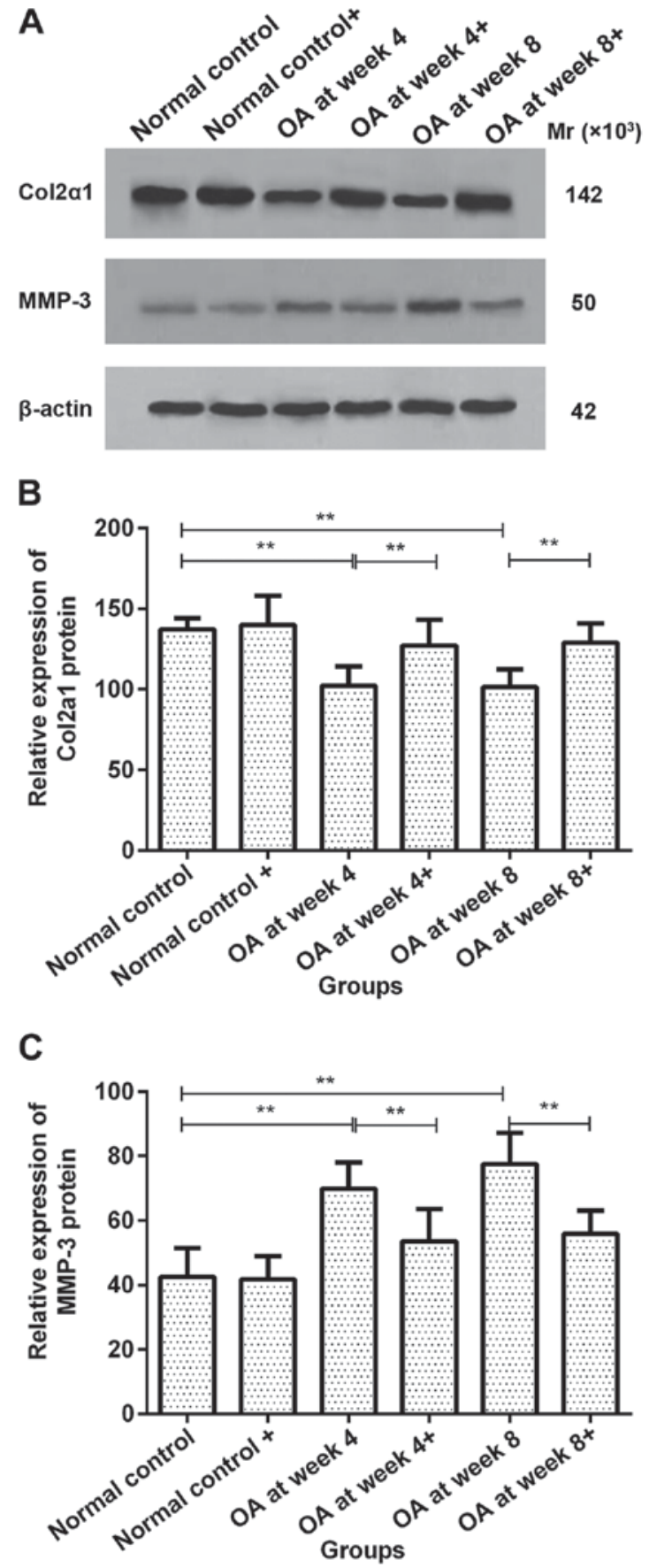

Figure 3. MMP-3 and Col2a1 protein expressions were assessed in chondrocytes using western blotting. + indicates chondrocytes following transfection. (A) MMP-3 and Col2a1 protein expressions using western blotting. (B) The expression of Col2al protein increased at week 4 and 8 after transfection $\left({ }^{* *} \mathrm{P}<0.01\right)$. (C) The expression of MMP-3 protein decreased at week 4 and 8 after transfection $\left({ }^{* * *} \mathrm{P}<0.01\right)$. Data are presented as the mean \pm standard error of the mean. $n=10 .{ }^{* *} \mathrm{P}<0.01$. MMP, metalloproteinase; OA, osteoarthritis.

mRNA expression was observed in normal control group. However, Col2a1 mRNA levels in groups OA at week 4 and 8 were increased by 63 and $126 \%$, respectively, following transfection ( $\mathrm{P}<0.01$; Fig. 2). Furthermore, no significant difference in Col2a1 expression was identified between groups OA at week 4 and 8 following transfection.

Col2al protein expression in chondrocytes prior to and following ds-miRNA-4784 transfection. No significant 


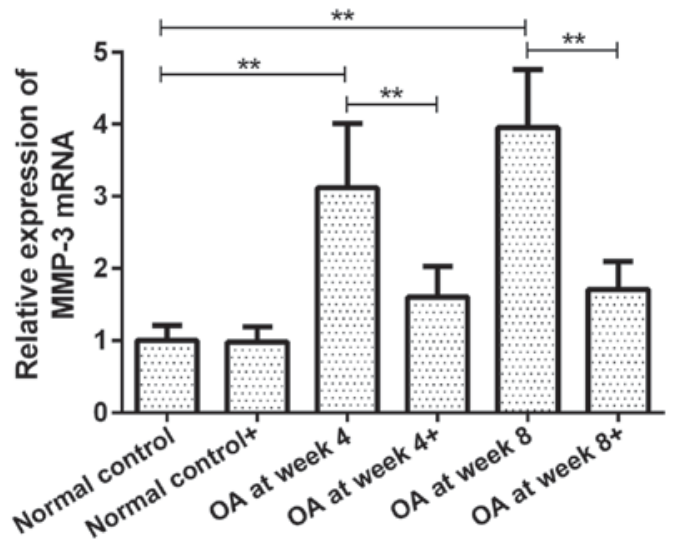

Figure 4. MMP-3 mRNA expression in chondrocytes. + indicates chondrocytes following transfection. Data are presented as the mean \pm standard error of the mean. $n=10 .{ }^{* *} \mathrm{P}<0.01$. MMP, metalloproteinase; OA, osteoarthritis.

difference in Col2al protein expression was observed in normal control group prior to and following ds-miRNA-4784 transfection. However, Col2a1 protein expression increased significantly in groups OA at week 4 and 8 following transfection compared with pre-transfection levels $(\mathrm{P}<0.01$; Fig. 3A and B).

MMP-3 protein expression in chondrocytes prior to and following ds-miRNA-4784 transfection. No significant difference in MMP-3 protein expression was observed in normal control group prior to and following ds-miRNA-4784 transfection. However, the expression of MMP-3 protein significantly decreased following transfection compared with pre-transfection levels in groups $\mathrm{OA}$ at week 4 and $8(\mathrm{P}<0.01$; Fig. 3A and C).

MMP-3 mRNA expression in chondrocytes prior to and following ds-miRNA-4784 transfection. The expression of MMP-3 mRNA in groups OA at week 4 and 8 prior to transfection were 3.12 and 3.95-fold higher than that in normal control group ( $\mathrm{P}<0.01$, Fig. 4). However, no significant difference was observed between groups $\mathrm{OA}$ at week 4 and 8. Following transfection with ds-miRNA-4784, no significant difference in MMP-3 mRNA expression was observed in normal control group compared with pre-transfection level. Furthermore, the expression of MMP-3 mRNA in groups OA at week 4 and 8 was significantly decreased following transfection compared with pre-transfection levels $(\mathrm{P}<0.01$; Fig. 4). No significant difference in MMP-3 mRNA expression was observed between groups $\mathrm{OA}$ at week 4 and 8 following transfection.

\section{Discussion}

OA is one of the most common types of chronic arthritis and seriously affects the quality of life in the elderly population (1-3). Major pathological features of OA include a series of biological and/or morphological changes, such as the degeneration of articular cartilage, hyperosteogeny and/or sclerosis (16). Currently, OA is typically treated conservatively, with the main aim being the pain relief; however, effective radical treatment remains insufficient (17). Furthermore, certain patients may experience disease recurrence due to ineffective treatment (18)

The occurrence of OA is associated with many parameters, including genetic and immune factors; however, the exact pathogenesis remains unclear (3). The roles of various miRNAs in OA chondrocytes have been widely studied and have provided a novel research direction, which has helped to elucidate the pathogenesis of OA (13). miRNAs are a group of ubiquitous endogenous small single-stranded non-coding RNAs that are associated with the regulation of $30 \%$ of genes (19). The abnormal expression of various miRNAs, including miRNA-140, -126 and -146 , has been observed in patients with OA $(13,14)$. These miRNAs advance the development of OA by promoting the expression of MMPs and reducing the mechanism of collagen (20).

In the present study, a rabbit OA model was established to assess the changes in miRNA-4784 expression in chondrocytes at 4 and 8 weeks following the model construction. The results revealed that the expression of miRNA-4784 in chondrocytes gradually decreased with prolonged duration of the disease, indicating that miRNA-4784 is downregulated in OA chondrocytes. However, the mechanism by which this occurs remains unclear. Previous studies have revealed that the expression of a series of miRNAs, including miRNA-140, decrease in chondrocytes during early OA; this change may be associated with miRNA inhibition by cytokines such as metalloproteinases or collagen $(13,20)$.

Changes in the expression of Col2al and MMP-3 in OA chondrocytes were also assessed in the present study. Col2a1 expression serves an important role in maintaining the normal function of chondrocytes, the downregulation of which indicates the degeneration of articular cartilage (21-23). MMP-3 is an important chondroitin-degrading enzyme whose upregulation promotes the degeneration of articular cartilage (24). In the present study, compared with normal control group, the expression of Col2a1 in OA chondrocytes was significantly reduced at the miRNA and protein levels, which is consistent with the results of previous studies (25-27). Furthermore, an increase in MMP-3 expression was observed in OA chondrocytes, indicating that there was a degenerative change in the cartilage during early stage OA. Additionally, miRNA-4784 levels increased following transfection with exogenous ds-miRNA-4784, while Col2a1 expression also increased in OA chondrocytes. The expression of MMP-3 mRNA and protein were decreased, suggesting that ds-miRNA-4784 transfection improves the function of OA chondrocytes.

The results of the present study also demonstrated that miRNA-4784 expression gradually decreases with prolonged disease duration. Transfection-induced miRNA-4784 upregulation maintained the stability of chondrocytes, promoted the expression of $\mathrm{Col} 2 \mathrm{a} 1$ and inhibited the expression of MMP-3. These results confirm that miRNA-4784 serves a role in the development and progression of OA. In this investigation, we only studied the mechanism of miRNA-4784 in the pathogenesis of OA from experimental animals, but we did not detect the cytokines and RNA in clinical patients. Therefore, more research is needed to further confirm the role of miRNA-4784 in the pathogenesis of OA. The present study may provide novel insights into the pathogenesis of $\mathrm{OA}$ and a theoretical basis for the application of gene therapy in future treatments for OA. 


\section{Acknowledgements}

Not applicable.

\section{Funding}

No funding was received.

\section{Availability of data and materials}

The datasets used and/or analyzed during the current study are available from the corresponding author on reasonable request.

\section{Authors' contributions}

JL and QY were major contributors in writing the manuscript and participated in the analysis and discussion of the data. JL and YYa were responsible for the cell culture. XC and YYe performed RT-qPCR and western blotting. All authors read and approved the final version of the manuscript.

\section{Ethics approval and consent to participate}

The present study was approved by the Ethics Committee of the 174th Hospital of Chinese PLA (Chenggong Hospital Affiliated to Medical College of Xiamen University, Xiamen, China).

\section{Patient consent for publication}

Not applicable.

\section{Competing interests}

The authors declare that they have no competing interests.

\section{References}

1. Taheri P, Vahdatpour B, Asl MM and Ramezanian H: Effects of taping on pain and functional outcome of patients with knee osteoarthritis: A pilot randomized single-blind clinical trial. Adv Biomed Res 6: 139, 2017.

2. Brazilian Medical Association, Silvinato A and Bernardo WM: Inflammatory arthritis or osteoarthritis of the knee - efficacy of intra-joint infiltration of methylprednisolone acetate versus triamcinolone acetonide or triamcinolone hexacetonide. Rev Assoc Med Bras (1992) 63: 827-836, 2017.

3. Yang M, Jiang L, Wang Q, Chen $\mathrm{H}$ and Xu G: Traditional Chinese medicine for knee osteoarthritis: An overview of systematic review. PLoS One 12: e0189884, 2017.

4. Whitney KE, Liebowitz A, Bolia IK, Chahla J, Ravuri S, Evans TA, Philippon MJ and Huard J: Current perspectives on biological approaches for osteoarthritis. Ann NY Acad Sci 1410: 26-43, 2017.

5. Nielsen FK, Egund N, Jørgensen A and Jurik AG: Risk factors for joint replacement in knee osteoarthritis; a 15-year follow-up study. BMC Musculoskelet Disord 18: 510, 2017.

6. Duarte N, Rodrigues AM, Branco JDC, Canhão H, Hughes SL and Paúl C: Health and lifestyles factors associated with osteoarthritis among older adults in Portugal. Front Med (Lausanne) 4: $192,2017$.

7. Luo S, Shi Q, Chen J, Wang H, Wu W and Zha Z: Expression and significance of MMPs in synovial fluid, serum and PBMC culture supernatant stimulated by LPS in osteoarthritis patients with or without diabetes. Exp Clin Endocrinol Diabetes: Dec 21, 2017 (Epub ahead of print).

8. Miyaki S and Asahara H: Macro view of microRNA function in osteoarthritis. Nat Rev Rheumatol 8: 543-552, 2012.
9. Barlas IO, Sezgin M, Erdal ME, Sahin G, Ankarali HC, Altintas ZM and Türkmen E: Association of $(-1,607) 1 \mathrm{G} / 2 \mathrm{G}$ polymorphism of matrix metalloproteinase-1 gene with knee osteoarthritis in the Turkish population (knee osteoarthritis and MMPs gene polymorphisms). Rheumatol Int 29: 383-388, 2009.

10. Akhtar N, Khan NM, Ashruf OS and Haqqi TM: Inhibition of cartilage degradation and suppression of PGE2 and MMPs expression by pomegranate fruit extract in a model of posttraumatic osteoarthritis. Nutrition 33: 1-13, 2017.

11. Xu P, Yao J and Hou W: Relationships between COL2A1 gene polymorphisms and knee osteoarthritis in Han Chinese women. Mol Biol Rep 38: 2377-2381, 2011.

12. Tao L, Zeng Y, Wang J, Liu Z, Shen B, Ge J, Liu Y, Guo Y and Qiu J: Differential microRNA expression in aristolochic acid-induced upper urothelial tract cancers ex vivo. Mol Med Rep 12: 6533-6546, 2015.

13. Zhen Y, Xinghui Z, Chao W, Yi Z, Jinwen C, Ruifang G, Chao Z, Min Z, Chunlei G, Yan F, et al: Several microRNAs could predict survival in patients with hepatitis B-related liver cancer. Sci Rep 7: 45195, 2017.

14. Soyocak A, Kurt H, Ozgen M, Turgut Cosan D, Colak E and Gunes HV: miRNA-146a, miRNA-155 and JNK expression levels in peripheral blood mononuclear cells according to grade of knee osteoarthritis. Gene 627: 207-211, 2017.

15. Livak KJ and Schmittgen TD: Analysis of relative gene expression data using real-time quantitative PCR and the 2(-Delta Delta C(T)) method. Methods 25: 402-408, 2001.

16. Brisson NM, Stratford PW and Maly MR: Relative and absolute test-retest reliabilities of biomechanical risk factors for knee osteoarthritis progression: Benchmarks for meaningful change. Osteoarthritis Cartilage 26: 220-226, 2018.

17. Abbate LM, Jeffreys AS, Coffman CJ, Schwartz TA, Arbeeva L, Callahan LF, Negbenebor NA, Kohrt WM, Schwartz RS, Vina E, et al: Demographic and clinical factors associated with nonsurgical osteoarthritis treatment use among patients in outpatient clinics. Arthritis Care Res (Hoboken) 70: 1141-1149, 2018.

18. Snochowska A, Szmigielska P, Brzeziańska-Lasota E and Tomaszewski W: Genetic and epigenetic interactions in the etiopathogenesis of osteoarthritis. Selected molecular factors in OA etiopathogenesis. Ortop Traumatol Rehabil 19: 227-237, 2017.

19. Kopańska M, Szala D, Czech J, Gabło N, Gargasz K, Trzeciak M, Zawlik I and Snela S: MiRNA expression in the cartilage of patients with osteoarthritis. J Orthop Surg Res 12: 51, 2017.

20. Nugent M: MicroRNAs: Exploring new horizons in osteoarthritis. Osteoarthritis Cartilage 24: 573-580, 2016.

21. Bai Y, Kong X, Liu N, Ren S, Guo H and Zhao K: Mutational analysis and prenatal diagnosis of COL1A1 and COL1A2 genes in four Chinese families affected with osteogenesis imperfecta. Zhonghua Yi Xue Yi Chuan Xue Za Zhi 34: 705-708, 2017 (In Chinese).

22. Loughlin J, Sinsheimer JS, Mustafa Z, Carr AJ, Clipsham K, Bloomfield VA, Chitnavis J, Bailey A, Sykes B and Chapman K: Association analysis of the vitamin D receptor gene, the type I collagen gene COL1A1, and the estrogen receptor gene in idiopathic osteoarthritis. J Rheumatol 27: 779-784, 2000.

23. Aerssens J, Dequeker J, Peeters J, Breemans S and Boonen S: Lack of association between osteoarthritis of the hip and gene polymorphisms of VDR, COL1A1, and COL2A1 in postmenopausal women. Arthritis Rheum 41: 1946-1950, 1998.

24. Tong Z, Liu Y, Chen B, Yan L and Hao D: Association between MMP3 and TIMP3 polymorphisms and risk of osteoarthritis. Oncotarget 8: 83563-83569, 2017.

25. Shi J, Zhang C, Yi Z and Lan C: Explore the variation of MMP3, JNK, p38 MAPKs, and autophagy at the early stage of osteoarthritis. IUBMB Life 68: 293-302, 2016.

26. Chen JJ, Huang JF, Du WX and Tong PJ: Expression and significance of MMP3 in synovium of knee joint at different stage in osteoarthritis patients. Asian Pac J Trop Med 7: 297-300, 2014.

27. Kubota E, Imamura H, Kubota T, Shibata T and Murakami K: Interleukin 1 beta and stromelysin (MMP3) activity of synovial fluid as possible markers of osteoarthritis in the temporomandibular joint. J Oral Maxillofac Surg 55: 20-28, 1997.

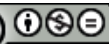

This work is licensed under a Creative Commons Attribution-NonCommercial-NoDerivatives 4.0 International (CC BY-NC-ND 4.0) License. 